Pacific Journal of Mathematics

NONLINEAR REPRESENTATIONS OF POINCARÉ GROUP AND Q QUES C. H. SIMON 


\title{
NON-LINEAR REPRESENTATIONS OF POINCARÉ GROUP AND GLOBAL SOLUTIONS OF RELATIVISTIC WAVE EQUATIONS
}

\author{
JACQUeS C. H. SimON
}

\begin{abstract}
Non-linear massive representations of the Poincare group are proved to be equivalent, on certain sectors, to massive linear representations with an energy of definite sign. As a consequence (for small initial data in these sectors), the existence of global solutions for massive wave equations is proved.
\end{abstract}

1. Introduction. The aim of this article is to study a family of non-linear representations of the Poincare group $P=\operatorname{SL}(2, C) \cdot T_{4}$, the universal covering of the inhomogeneous Lorentz group. By non-linear representation of a real Lie group one means a non-linear local action, in a vector space, which has a fixed point (say the origin).

Non-linear representations of the Poincare group appear in a natural way in the study of relativistic wave equations, where the one parameter group of evolution is imbedded in a non-linear representation of the Poincaré group.

A formal study of this aspect of relativistic wave equations can be found in references [2-5]. The main results, there, are that the evolution of a massless wave equation is intertwined by a formal power series with the evolution of the corresponding free wave equation, and that this is also true for the evolution of a massive equation in some sectors of the space of initial conditions.

One now proves that, for massive fields, this intertwining series is convergent in some domains which will be explicated later. The proof steps will seem natural to those who are familiar with linearization, without small denominators, of vector fields [9]:

1. Check that there is no cohomological obstruction (no resonance condition on the eigenvalues of the linear part, in the language of vector fields) in order to prove the existence of the intertwining formal power series.

In the present situation this is obtained by extending the calculus to the enveloping algebra of $P$ where the existence of a resolvent for the mass operator in a tensor product of representations permits to trivialize the cohomology. The study of the resolvent of the mass operator is done in part 3 and the construction of the formal intertwining in part 4. 
2. Prove the convergence of the intertwining series. For vector fields this is easily obtained if there are no small denominators. In the present situation, the analogous condition is a bound for the norm of resolvent of the mass operator in a tensor product of representations. Convergence of the intertwining series is proved in part 5.

The intertwining power series between the time evolution of the non-linear wave equation and the evolution of the corresponding free field can be viewed as an abstract wave operator the existence of which, in some sectors, implies the existence of global solutions.

The wave equation $\square \varphi+m^{2} \varphi=J(\varphi), J$ analytic around the origin and $J(0)=J^{\prime}(0)=0$, is given in part 6 as an example of a wave equation with (non-zero) global solutions for small initial data in some sectors. Though the formulation is given in a four-dimensional space-time, the results are not sensitive to space-time dimension.

The author wishes to thank Professor M. Flato for interesting discussions and valuable information, and Dr. D. Sternheimer for careful reading of the manuscript.

2. Notations. Suppose given a Lorentz basis $\left(X_{0}, X_{1}, X_{2}, X_{3}\right)$, with $X_{0}$ time-like, in the dual space $T_{4}^{*}$ of $T_{4}$. If $p \in T_{4}^{*}, p=\Sigma_{\mu=0}^{3} p^{\mu} X_{\mu}$ one uses the notation $p=\left(p^{0}, \vec{p}\right)$ with $\vec{p}=\Sigma_{J=1}^{3} p^{J} X_{j}$.

If $m \geq 0$ and $\varepsilon= \pm 1$ one defines the surface

$$
M(m, \varepsilon)=\left\{p=\left.\sum_{\mu=0}^{3} p^{\mu} X_{\mu}\left|(p)^{2}=\left(p^{0}\right)^{2}-\right| \vec{p}\right|^{2}=m^{2}, \varepsilon p^{0}>0\right\} .
$$

One denotes by $d \nu(p)=d \vec{p} /\left|p^{0}\right|$ the invariant measure on $M(m, \varepsilon)$. Given a unitary representation $(V, \Sigma)$ of the stabilizer $H$ of a point $p \in M(m, \varepsilon)$ in a Hilbert space $\Sigma$, one denotes by $U^{m, \varepsilon}$ the representation of $P$ induced by $V$ on the space $E(M(m, \varepsilon))=L_{d \nu}^{q}(M(m, \varepsilon), \Sigma)$. In what follows $q=1$ or 2 . On $T_{4}$ the representation writes

$$
\left(U_{g}^{m, \varepsilon} f\right)(p)=e^{i\langle g, p\rangle} f(p) \text { and }\left(d U_{x}^{m, \varepsilon} f\right)(p)=i\langle x, p\rangle f(p),
$$

where $f \in E(M(m, \varepsilon)), p \in M(m, \varepsilon), g \in T_{4}, x \in \mathfrak{t}_{4}$ (the Lie algebra of $\left.T_{4}\right)$. $U^{m, \varepsilon}$ is norm preserving on $E(M(m, \varepsilon))$. One denotes by $E_{\infty}(M(m, \varepsilon))$ (resp. $E_{\tau}(M(m, \varepsilon))$ ) the space of $C^{\infty}$ vectors of $U^{m, \varepsilon}$ (resp. $\left.U^{m, \varepsilon}\right|_{T_{4}}$ ). Given a compact set $K \subset M(m, \varepsilon)$, one denotes by $E(K)$ the space of functions in $L_{d \nu}^{q}(M(m, \varepsilon), \Sigma)$ with support included in $K$; obviously $E(K) \subset E_{\tau}(M(m, \varepsilon))$. Denote by $E_{c}(M(m, \varepsilon))=\bigsqcup_{K} E(K)$ the union being taken over all $K$, compact, in $M(m, \varepsilon)$.

Suppose that $m>0$ and $p \in M(m, \varepsilon)$; one denotes by $\Lambda(p)$ the set of the Lorentz bases for which $\vec{p}=0$; if $K$ is compact in $M(m, \varepsilon)$, define $\Lambda(K) \cup_{p \in K} \Lambda(p)$ which can be identified with a compact subset of $S O(1,3)$. 
Suppose that $m_{1}>0$ and $m_{2} \geq 0 ; K_{1}$ (resp. $K_{2}$ ) being a compact subset of $M\left(m_{1}, \varepsilon\right)\left(\right.$ resp. $\left.M_{2}\left(m_{2}, \varepsilon\right)\right)$, define

$$
c\left(K_{1}, K_{2}\right)=\inf _{p \in K_{2}, L \in \Lambda\left(K_{1}\right)}\left(\varepsilon p^{0}\right)
$$

and

$$
d\left(K_{1}, K_{2}\right)=\inf _{p \in K_{2}, L \in \Lambda\left(K_{1}\right)}\left(\varepsilon p^{0}-|\vec{p}|\right)
$$

Given two topological vector spaces $Y, Z$, one denotes by $\mathfrak{L}_{n}(Y, Z)$ the space of continuous $n$-linear symmetric mappings from $Y^{n}$ to $Z$ and by $\mathfrak{F}(Y, Z)$ the space of formal power series from $Y$ to $Z$ of the form $f=\Sigma_{n \geq 1} f^{n}, f^{n} \in \mathfrak{L}_{n}(Y, Z)$. When $Y$ and $Z$ are Banach spaces and $\lambda>0$, $\mathfrak{S}_{\lambda}(Y, Z)$ is the Banach space of formal power series $f \in \mathfrak{F}(Y, Z)$ such that $\|f\|=\Sigma_{n \geq 1} \lambda^{n}\left\|f^{n}\right\|<+\infty$.

Given two topological vector spaces $Y, Z, A \in \mathfrak{F}(Y, Z)$, and $B \in$ $\mathfrak{F}(Y, Y)$, one defines $A * B \in \mathfrak{F}(Y, Z)$ by

$$
A * B=\sum_{n \geq 1}\left(\sum_{1 \leq p \leq n} A^{p}\left(\sum_{0 \leq q \leq p-1} I_{q} \otimes B^{n-p+1} \otimes I_{p-q-1}\right) \sigma_{n}\right)
$$

where $I_{q}$ is the identity mapping on $X \hat{\otimes}_{\pi} \cdots \hat{\otimes}_{\pi} X\left(q\right.$ times) and $\sigma_{n}$ is the symmetrization operator on $X \hat{\otimes}_{\pi} \cdots \hat{\otimes}_{\pi} X$ ( $n$ times):

$$
\sigma_{n}\left(\varphi_{1} \otimes \cdots \otimes \varphi_{n}\right)=\frac{1}{n !} \sum_{\sigma \in \Phi_{n}} \varphi_{\sigma(1)} \otimes \cdots \otimes \varphi_{\sigma(n)},
$$

$\mathfrak{S}_{n}$ being the group of permutations of $n$ elements. Whenever $Y=Z$ one defines $[A, B]_{*}=A * B-B * A$. Given two Banach spaces $X$ and $Y$, the norm on $X \otimes_{\pi} Y$ is defined by $\|z\|=\inf \Sigma_{i, j}\left|a_{i j}\right|$, the infimum being taken over all the ways of writing $z=\sum_{i, j} a_{i j} x_{i} y_{j}$ with $\left\|x_{i}\right\|=\left\|y_{j}\right\|=1$. In general we denote by $X \hat{\otimes}_{\pi} Y$ the completed projective tensor product of two locally convex topological vector spaces $X$ and $Y$.

3. Resolvent of the mass operator. Denote by $(U, \mathfrak{X})$ the linear representation of the Poincare group defined on the Banach space $\mathfrak{X}=$ $E\left(M\left(m_{1}, \varepsilon\right)\right) \hat{\otimes}_{\pi} \cdots \hat{\otimes}_{\pi} E\left(M\left(m_{n}, \varepsilon\right)\right)$ by $U=U^{m_{1}, \varepsilon} \hat{\otimes}_{\pi} \cdots \hat{\otimes}_{\pi} U^{m_{n}, \varepsilon}$. Choose a Lorentz basis $L=\left(X_{0}, X_{1}, X_{2}, X_{3}\right)$ of $\mathrm{t}_{4}$. The mass operator $Q\left(m_{1}, \ldots, m_{n}, \varepsilon\right)$ is defined on the space $D_{Q}=E_{\infty}\left(M\left(m_{1}, \varepsilon\right)\right) \hat{\otimes}_{\pi}$ $\cdots \hat{\otimes}_{\pi} E_{\infty}\left(M\left(m_{n}, \varepsilon\right)\right)$ by

$$
Q\left(m_{1}, \ldots, m_{n}, \varepsilon\right)=-\left(d U_{X_{0}}\right)^{2}+\sum_{j=1}^{3}\left(d U_{X_{j}}\right)^{2}
$$


$D_{Q}$ is a space of $C^{\infty}$ functions from $\prod_{j=1}^{n} M\left(m_{j}, \varepsilon\right)$ to $\Sigma_{1} \hat{\otimes}_{\pi} \cdots \hat{\otimes}_{\pi} \Sigma_{n}$. Therefore if $f \in D_{Q}$, we have

$$
\left(Q\left(m_{1}, \ldots, m_{n}, \varepsilon\right) f\right)\left(p_{1}, \ldots, p_{n}\right)=\left(p_{1}+\cdots+p_{n}\right)^{2} f\left(p_{1}, \ldots, p_{n}\right)
$$

where $p_{j} \in M\left(m_{j}, \varepsilon\right)$. The operator $Q\left(m_{1}, \ldots, m_{n}, \varepsilon\right)$ is obviously independent of the choice of the Lorentz basis.

Proposition 3.1. Suppose that $1 \leq k \leq n, m_{1} \neq 0, \ldots, m_{k} \neq 0$, $m_{k+1}=\cdots=0$. Given $K_{r}$, compact, in $M\left(m_{r}, \varepsilon\right)(r=1, \ldots, n)$, define $E_{\infty}\left(K_{r}\right)=E\left(K_{r}\right) \cap E_{\infty}\left(M\left(m_{r}, \varepsilon\right)\right)$. The restriction $Q^{\prime}\left(m_{1}, \ldots, m_{n}, \varepsilon\right)$ of $Q\left(m_{1}, \ldots, m_{n}, \varepsilon\right)$ to $D_{Q^{\prime}}=E_{\infty}\left(K_{1}\right) \hat{\otimes}_{\pi} \ldots \hat{\otimes}_{\pi} E_{\infty}\left(K_{n}\right)$ is closable and has $a$ resolvent $R_{\lambda}=\left(\bar{Q}^{\prime}\left(m_{1}, \ldots, m_{n}, \varepsilon\right)-\lambda\right)^{-1}$ in the Banach space

$$
\begin{aligned}
& E\left(K_{1}\right) \hat{\otimes}_{\pi} \cdots \hat{\otimes}_{\pi} E\left(K_{n}\right) \\
& \text { for } \lambda<2\left(\sum_{1 \leq i \leq k} d\left(K_{1}, K_{i}\right)\right)\left(\sum_{k+1 \leq j \leq n} c\left(K_{1}, K_{j}\right)\right)+\left(m_{1}+\cdots+m_{n}\right)^{2} .
\end{aligned}
$$

Moreover

$$
\begin{array}{r}
\left\|R_{\lambda}\right\| \leq\left(2\left(\sum_{1 \leq i \leq k} d\left(K_{1}, K_{i}\right)\right)\left(\sum_{k+1 \leq j \leq n} c\left(K_{1}, K_{J}\right)\right)\right. \\
\left.+\left(m_{1}+\cdots+m_{n}\right)^{2}-\lambda\right)^{-1}
\end{array}
$$

Proof. Suppose, say, that $\varepsilon=+1$. Write for short $Q^{\prime}=$ $Q^{\prime}\left(m_{1}, \ldots, m_{n}, \varepsilon\right)$.

(a) Suppose that $E\left(M\left(m_{i}, \varepsilon\right)\right)=L_{d \nu}^{1}\left(M\left(m_{i}, \varepsilon\right), \Sigma_{i}\right)$. Take $p_{r} \in K_{r}$ $(r=1, \ldots, n)$. Then $\left(p_{1}+\cdots+p_{n}\right)^{2}-\lambda=2 \sum_{i<j} p_{i} \cdot p_{j}+\sum_{i=1}^{n} m_{i}^{2}-\lambda$.

Since $p_{i} \cdot p_{j} \geq m_{i} m_{j}$ we have

$$
\begin{aligned}
\left(p_{1}+\cdots+p_{n}\right)^{2}-\lambda \geq & 2\left(\sum_{1 \leq i \leq k} p_{i}\right)\left(\sum_{k+1 \leq j \leq n} p_{j}\right) \\
& +\left(m_{1}+\cdots+m_{n}\right)^{2}-\lambda .
\end{aligned}
$$

If $m_{j}=0$, expressing the vectors in a Lorentz basis in $\Lambda\left(K_{1}\right)$, we have

$$
p_{i} \cdot p_{j} \geq\left(p_{i}^{0}-\left|\vec{p}_{i}\right|\right) p_{j}^{0} \geq d\left(K_{1}, K_{i}\right) c\left(K_{1}, K_{j}\right) \text {. }
$$

Therefore

$$
\begin{aligned}
\left(p_{1}+\cdots+p_{n}\right)^{2}-\lambda \geq & 2\left(\sum_{1 \leq i \leq k} d\left(K_{1}, K_{i}\right)\right)\left(\sum_{k+1 \leq j \leq n} c\left(K_{1}, K_{j}\right)\right) \\
& +\left(m_{1}+\cdots+m_{n}\right)^{2}-\lambda .
\end{aligned}
$$


Denote by $Z=E\left(K_{1}\right) \hat{\otimes}_{\pi} \cdots \hat{\otimes}_{\pi} E\left(K_{n}\right)$. It is known [6, §2] that $Z$ is isomorphic to $L^{1}\left(K_{1} \times \cdots \times K_{n}, \Sigma_{1} \hat{\otimes}_{\pi} \cdots \hat{\otimes}_{\pi} \Sigma_{n}\right)$ (the measure on $K_{1} \times \cdots \times K_{n}$ being the tensor product measure).

If one defines $R_{\lambda}$ on $Z$ by

$$
\left(R_{\lambda} f\right)\left(p_{1}, \ldots, p_{n}\right)=\left(\left(p_{1}+\cdots+p_{n}\right)^{2}-\lambda\right)^{-1} f\left(p_{1}, \ldots, p_{n}\right),
$$

inequality (3.1) is satisfied.

Take $\varphi \in D_{Q^{\prime}}$; we have $R_{\lambda}\left(Q^{\prime}-\lambda\right) \varphi=\varphi$. Take a sequence $\varphi_{k} \in D_{Q^{\prime}}$ converging to 0 and such that $\left(Q^{\prime}-\lambda\right) \varphi_{k}$ converges to a limit $\psi$ in $Z^{\prime}$. Then, $\varphi_{l}=R_{\lambda}\left(Q^{\prime}-\lambda\right) \varphi_{i}$ converges to $R_{\lambda} \psi$. Therefore $R_{\lambda} \psi=0$ and $\psi=0$. This means that $Q^{\prime}-\lambda$ is closable and so is $Q^{\prime}$.

Take now any $\psi \in Z$ and $\varphi=R_{\lambda} \psi$ and choose a sequence $\psi_{k} \in D_{Q^{\prime}}$ which converges to $\psi$. The sequence $R_{\lambda} \psi_{k}$ converges to $\varphi$; the relation $\psi_{i}=\left(Q^{\prime}-\lambda\right) R_{\lambda} \psi_{i}$ implies that $\varphi$ is in the domain of $\overline{Q^{\prime}}$ and that $\left(\overline{Q^{\prime}}-\lambda\right) R_{\lambda}=\operatorname{Id}_{Z}$.

(b) Suppose that $E\left(M\left(m_{i}, \varepsilon\right)\right)=L_{d \nu}^{2}\left(M\left(m_{i}, \varepsilon\right), \Sigma_{i}\right)$. The mapping $R_{\lambda}$ defined above is continuous from $Y=Y^{2}$ to $Z=Y^{1}$, with

$$
Y^{q}=L_{d \nu}^{q}\left(K_{1}, \Sigma_{1}\right) \hat{\otimes}_{\pi} \cdots \hat{\otimes}_{\pi} L_{d \nu}^{q}\left(K_{n}, \Sigma_{n}\right)
$$

In order to prove that $R_{\lambda}$ is continuous from $Y$ to itself one has to write the function $\mu\left(p_{1}, \ldots, p_{n}\right)=\left(\left(p_{1}+\cdots+p_{n}\right)^{2}-\lambda\right)^{-1}$ as a series of products of functions of one variable and then evaluate the norm in $Y$. Write $\sigma=\sum_{i=1}^{n} m_{i}^{2}-\lambda$; then $\mu\left(p_{1}, \ldots, p_{n}\right)=\left(\sigma+2 \Sigma_{i<j} p_{i} p_{j}\right)^{-1}$. Choose a Lorentz basis $L \in \Lambda\left(K_{1}\right)$, then

$$
\begin{aligned}
& \mu\left(p_{1}, \ldots, p_{n}\right) \\
& \quad=\frac{m_{1}}{p_{1}^{0}} \nu_{L}\left(p_{2}, \ldots, p_{n}\right)\left(1-\xi_{L}\left(p_{1}, \ldots, p_{n}\right) \nu_{L}\left(p_{2}, \ldots, p_{n}\right)\right)^{-1}
\end{aligned}
$$

where

$$
\nu_{L}\left(p_{2}, \ldots, p_{n}\right)=\left(\sigma+2 m_{1} \sum_{j>1} p_{j}^{0}+2 \sum_{1<i<j} p_{i} \cdot p_{j}\right)^{-1}
$$

and

$$
\begin{aligned}
\xi_{L}\left(p_{1}, \ldots, p_{n}\right)= & \left(1-\frac{m_{1}}{p_{1}^{0}}\right)\left(\sigma+2 \sum_{1<i<j} p_{i} \cdot p_{j}\right) \\
& +2 m_{1} \frac{\vec{p}_{1}}{p_{1}^{0}} \cdot \sum_{j>1} \vec{p}_{J} .
\end{aligned}
$$


Noticing that $\left|\xi_{L}\left(p_{1}, \ldots, p_{n}\right)\right|<\left(\nu_{L}\left(p_{2}, \ldots, p_{n}\right)\right)^{-1}$, we can write

$$
\mu\left(p_{1}, \ldots, p_{n}\right)=\sum_{k \geq 1} \mu_{k, L}\left(p_{1}, \ldots, p_{n}\right)
$$

with

$$
\text { (3.6) } \mu_{k, L}\left(p_{1}, \ldots, p_{n}\right)=\frac{m_{1}}{p_{1}^{0}}\left(\xi_{L}\left(p_{1}, \ldots, p_{n}\right)\right)^{k-1}\left(\nu_{L}\left(p_{2}, \ldots, p_{n}\right)\right)^{k}
$$

Define

$$
\begin{aligned}
\rho= & 2\left(\sum_{1 \leq i \leq k} d\left(K_{1}, K_{i}\right)\right)\left(\sum_{k+1 \leq j \leq n} c\left(K_{1}, K_{j}\right)\right) \\
& +\left(m_{1}+\cdots+m_{n}\right)^{2}-\lambda .
\end{aligned}
$$

If $m_{i} \neq 0$ we define $m_{i}^{\prime}=m_{i}$ and if $m_{i}=0$ we define $m_{i}^{\prime}=$ $\inf _{p \in K_{l}, L \in \Lambda\left(K_{1}\right)}\left(p^{0}\right)$. Define then

$$
\sigma^{\prime}=2\left(\sum_{1 \leq i \leq k} d\left(K_{1}, K_{i}\right)\right)\left(\sum_{k+1 \leq j \leq n} c\left(K_{1}, K_{j}\right)\right)+\sum_{i=1}^{n}\left(m_{i}^{\prime}\right)^{2}-\lambda
$$

and

$\rho^{\prime}=2\left(\sum_{1 \leq i \leq k} d\left(K_{1}, K_{i}\right)\right)\left(\sum_{k+1 \leq j \leq n} c\left(K_{1}, K_{j}\right)\right)+\left(m_{1}^{\prime}+\cdots+m_{n}^{\prime}\right)^{2}-\lambda$.

Now,

$$
\nu_{L}\left(p_{2}, \ldots, p_{n}\right)=\frac{m_{2}^{\prime} \cdots m_{n}^{\prime}}{\rho^{\prime} p_{2}^{0} \cdots p_{n}^{0}}\left(1-\tau_{L}\left(p_{2}, \ldots, p_{n}\right)\right)^{-1}
$$

where

$$
\begin{aligned}
& \tau_{L}\left(p_{2}, \ldots, p_{n}\right) \\
& =\frac{1}{\rho^{\prime} p_{2}^{0} \cdots p_{n}^{0}}\left\{\sigma^{\prime}\left(\prod_{i>1} p_{i}^{0}-\prod_{i>1} m_{i}^{\prime}\right)+\left(\sigma^{\prime}-\sigma\right) \prod_{i>1} m_{i}^{\prime}\right. \\
& +2 m_{1} \sum_{j>1} m_{j}^{\prime} p_{j}^{0}\left(\prod_{i \neq 1, j} p_{i}^{0}-\prod_{i \neq 1, j} m_{i}^{\prime}\right) \\
& +2 \sum_{1<i<j} m_{i}^{\prime} m_{j}^{\prime} p_{i}^{0} p_{j}^{0}\left(\prod_{l \neq 1, i, j} p_{l}^{0}-\prod_{l \neq 1, i, j} m_{l}^{\prime}\right) \\
& \left.+2 m_{2}^{\prime} \cdots m_{n}^{\prime} \sum_{1<i<j} \vec{p}_{i} \cdot \vec{p}_{j}\right\}
\end{aligned}
$$


Moreover $\tau_{L}\left(p_{2}, \ldots, p_{n}\right)<1$. We can therefore write (3.8) as a series

$$
\nu_{L}\left(p_{2}, \ldots, p_{n}\right)=\frac{m_{2}^{\prime} \cdots m_{n}^{\prime}}{\rho^{\prime} p_{2}^{0} \cdots p_{n}^{0}} \sum_{k \geq 0}\left(\tau_{L}\left(p_{2}, \ldots, p_{n}\right)\right)^{k} .
$$

Introduce the variables

$$
x_{j}=1-\frac{m_{j}^{\prime}}{p_{j}^{0}}, \quad y_{j}=1+\frac{m_{j}^{\prime}}{p_{j}^{0}}, \quad \text { and } \quad z=\frac{m_{2}^{\prime} \cdots m_{n}^{\prime}}{\rho^{\prime} p_{2}^{0} \cdots p_{n}^{0}} \sum_{1<i<j} \vec{p}_{i} \cdot \vec{p}_{j}
$$

Note that $1-m_{i_{1}}^{\prime} \cdots m_{i_{k}}^{\prime} / p_{i_{1}}^{0} \cdots p_{i_{k}}^{0}$ is a polynomial with positive coefficients in the variables $x_{i_{1}}, \ldots, x_{i_{k}}, y_{i_{1}}, \ldots, y_{i_{k}}$. Therefore, it results from (3.9) that

$$
\tau_{L}\left(p_{2}, \ldots, p_{n}\right)=\Omega\left(x_{2}, \ldots, x_{n}, y_{2}, \ldots, y_{n}, z\right)
$$

$\Omega$ being a polynomial with positive coefficients in the variables $x_{2}, \ldots, x_{n}$, $y_{2}, \ldots, y_{n}, z$.

Before proceeding further one needs the following lemma.

LEMMA 3.2. Suppose given $m_{1} \geq 0, \ldots, m_{r} \geq 0$, positive integers $\left(k_{i j}\right)_{1 \leq i<j \leq r}, K_{i}$ compact in $M\left(m_{i}, \varepsilon\right)$, and $f_{i} \in L_{d \nu}^{2}\left(K_{i}, \Sigma_{i}\right)(i=1, \ldots, r)$. Define

$$
h\left(p_{1}, \ldots, p_{r}\right)=\prod_{1 \leq i<j \leq r}\left(\vec{p}_{i} \cdot \vec{p}_{j}\right)^{k_{\imath \jmath}}
$$

and

$$
h^{\prime}\left(p_{1}, \ldots, p_{r}\right)=\prod_{1 \leq i<j \leq r}\left(\left|\vec{p}_{i} \| \vec{p}_{j}\right|\right)^{k_{\iota j}}
$$

Then, in the Banach space $L^{2}\left(K_{1}, \Sigma_{1}\right) \hat{\otimes}_{\pi} \cdots \hat{\otimes}_{\pi} L^{2}\left(K_{r}, \Sigma_{r}\right)$, we have

$$
\left\|h \cdot f_{1} \otimes \cdots \otimes f_{r}\right\| \leq\left\|h^{\prime} \cdot f_{1} \otimes \cdots \otimes f_{r}\right\| .
$$

Proof. Suppose that $r=2$; write $k=k_{12}$.

$$
\begin{aligned}
\left\|h \cdot f_{1} \otimes f_{2}\right\| \leq & \sum_{\alpha_{1}, \ldots, \alpha_{k}=1}^{3}\left(\int_{K_{1}}\left|p_{1}^{\alpha_{1}} \cdots p_{1}^{\alpha_{k}} f_{1}\left(p_{1}\right)\right|^{2} d \nu\left(p_{1}\right)\right)^{1 / 2} \\
& \times\left(\int_{K_{2}}\left|p_{2}^{\alpha_{1}} \cdots p_{2}^{\alpha_{k}} f_{2}\left(p_{2}\right)\right|^{2} d \nu\left(p_{2}\right)\right)^{1 / 2} .
\end{aligned}
$$


Therefore

$$
\begin{aligned}
\left\|h \cdot f_{1} \otimes f_{2}\right\| \leq & \left(\int_{K_{1}} \sum_{\alpha_{1}, \ldots, \alpha_{k}=1}^{3}\left|p_{1}^{\alpha_{1}} \cdots p_{1}^{\alpha_{k}} f_{1}\left(p_{1}\right)\right|^{2} d \nu\left(p_{1}\right)\right)^{1 / 2} \\
& \times\left(\int_{K_{2} \alpha_{1}, \ldots, \alpha_{k}=1} \sum_{2}^{3}\left|p_{2}^{\alpha_{1}} \cdots p_{2}^{\alpha_{k}} f_{2}\left(p_{2}\right)\right|^{2} d \nu\left(p_{2}\right)\right)^{1 / 2} .
\end{aligned}
$$

We get by induction

$$
\begin{aligned}
\| h \cdot f_{1} & \otimes \cdots \otimes f_{r} \| \\
\leq & \prod_{1 \leq i<j \leq r}\left(\int_{K_{i}} \sum_{\alpha_{1}, \ldots, \alpha_{k_{i j}}=1}^{3}\left|p_{i}^{\alpha_{1}} \cdots p_{i}^{\alpha_{k_{i j}}} f_{i}\left(p_{i}\right)\right|^{2} d \nu\left(p_{i}\right)\right)^{1 / 2} \\
& \times\left(\int_{K_{J} \alpha_{1}, \ldots, \alpha_{k_{1 j}}=1} \sum_{j}^{3}\left|p_{j}^{\alpha_{1}} \cdots p_{j}^{\alpha_{k_{i j}}} f_{j}\left(p_{j}\right)\right|^{2} d \nu\left(p_{j}\right)\right)^{1 / 2}
\end{aligned}
$$

So,

$$
\begin{aligned}
\left\|h \cdot f_{1} \otimes \cdots \otimes f_{r}\right\| \leq & \prod_{1 \leq i<j \leq r}\left(\left.\left.\int_{K_{l}}|| \vec{p}_{i}\right|^{k_{\imath \jmath}} f_{i}\left(p_{i}\right)\right|^{2} d \nu\left(p_{i}\right)\right)^{1 / 2} \\
& \times\left(\left.\left.\int_{K_{j}}|| \vec{p}_{j}\right|^{k_{\imath \jmath}} f_{j}\left(p_{j}\right)\right|^{2} d \nu\left(p_{j}\right)\right)^{1 / 2}
\end{aligned}
$$

Note that the right-hand side of the last inequality is equal to $\left\|h^{\prime} f_{1} \otimes \cdots \otimes f_{r}\right\|$.

Coming back to the proof of Proposition 2.1, one introduces the following quantities

$$
\nu_{L}^{\prime}\left(p_{2}, \ldots, p_{n}\right)=\left(\sigma+2 m_{1} \sum_{j>1} p_{j}^{0}+2 \sum_{1<i<j}\left(p_{i}^{0} p_{j}^{0}-\left|\vec{p}_{i}\right|\left|\vec{p}_{j}\right|\right)\right)^{-1}
$$

and $\tau_{L}^{\prime}\left(p_{2}, \ldots, p_{n}\right)$ defined as $\tau_{L}\left(p_{2}, \ldots, p_{n}\right)$ in expression (3.9) with $\vec{p}_{i} \cdot \vec{p}_{j}$ replaced by $\left|\vec{p}_{i}\right|\left|\vec{p}_{j}\right|$. We have $\tau_{L}^{\prime}\left(p_{2}, \ldots, p_{n}\right)<1$. Therefore

$$
\nu_{L}^{\prime}\left(p_{2}, \ldots, p_{n}\right)=\frac{m_{2}^{\prime} \cdots m_{n}^{\prime}}{\rho^{\prime} p_{2}^{0} \cdots p_{n}^{0}}\left(1-\tau_{L}^{\prime}\left(p_{2}, \ldots, p_{n}\right)\right)^{-1} .
$$


Choose $\eta>0$. There exist finite partitions $\left(V_{\beta}^{i}\right)_{\beta \in B}$ of $K_{i}, i=1, \ldots, n$, by measurable subsets such that:

1. For every $\beta \in B$ there exists $L_{\beta} \in \Lambda\left(K_{1}\right)$ such that, expressing $p=\left(p^{0}, \vec{p}\right)$ in the basis $L_{\beta}$, we have $|\vec{p}| \leq \eta$ and $1-m_{1} / p^{0} \leq \eta$ for any $p \in V_{\beta}^{1}$.

2.

$$
\Omega\left(\bar{x}_{2}, \ldots, \bar{x}_{n}, \bar{y}_{2}, \ldots, \bar{y}_{n}, \bar{z}\right) \leq(1+\eta) \sup _{V_{\beta_{2}}^{2} \times \cdots \times V_{\beta_{n}}^{n}} \tau_{L_{\beta_{1}}}^{\prime}\left(p_{2}, \ldots, p_{n}\right),
$$

where

$$
\begin{aligned}
& \bar{x}_{i}=\sup _{V_{\beta_{i}}^{\prime}} x_{i}, \bar{y}_{i}=\sup _{V_{\beta_{i}}^{\prime}} y_{i}, \text { and } \\
& \bar{z}=\sup _{V_{\beta_{2}}^{2} \times \cdots \times V_{\beta_{n}}^{n}}\left(\frac{m_{2}^{\prime} \cdots m_{n}^{\prime}}{\rho^{\prime} p_{2}^{0} \cdots p_{n}^{0}} \sum_{1<i<j}\left|\vec{p}_{i}\right|\left|\vec{p}_{j}\right|\right)
\end{aligned}
$$

for any $\beta_{1}, \ldots, \beta_{n} \in B$.

3.

$$
(1+\eta) \inf _{V_{\beta_{2}}^{2} \times \cdots \times V_{\beta_{n}}^{n}} \tau_{L_{\beta_{1}}}^{\prime}\left(p_{2}, \ldots, p_{n}\right) \geq \sup _{V_{\beta_{2}}^{2} \times \cdots \times V_{\beta_{n}}^{n}} \tau_{L_{\beta_{1}}^{\prime}}^{\prime}\left(p_{2}, \ldots, p_{n}\right)
$$

for any $\beta_{1}, \ldots, \beta_{n} \in B$.

4.

$$
(1+\eta) \inf _{V_{\beta_{i}}^{\prime}} p_{i}^{0} \geq \sup _{V_{\beta_{i}}^{i}} p_{i}^{0}
$$

for $\beta_{2}, \ldots, \beta_{n} \in B$ and any basis $L \in \Lambda\left(K_{1}\right)$.

Denote by $\chi_{\beta}^{i}$ the characteristic function of $V_{\beta}^{i}$. Take $f_{j} \in L_{d \nu}^{2}\left(K_{j}, \Sigma_{j}\right)$, $j=1, \ldots, n$. Taking into account (3.6), (3.10), (3.11), and Lemma 3.2, we have

$$
\begin{aligned}
& \left\|\mu_{k, L_{\beta_{1}}} \chi_{\beta_{1}}^{1} f_{1} \otimes \cdots \otimes \chi_{\beta_{n}}^{n} f_{n}\right\| \\
& \quad \leq C\left(k, \beta_{1}, \ldots, \beta_{n}\right)\left\|\chi_{\beta_{1}} f_{1}\right\| \cdots\left\|\chi_{\beta_{n}} f_{n}\right\|,
\end{aligned}
$$

where

$$
\begin{aligned}
& C\left(k, \beta_{1}, \ldots, \beta_{n}\right) \\
& =\eta^{k-1}\left(\sigma+2 \sum_{1<i<j}\left(\left(\sup _{K_{\imath}} p_{\imath}^{0}\right)\left(\sup _{K_{j}} p_{j}^{0}\right)\right.\right. \\
& \left.\left.\quad+\left(\sup _{K_{\imath}}\left|\vec{p}_{i}\right|\right)\left(\sup _{K_{j}}\left|\vec{p}_{j}\right|\right)+2 \sum_{j>1} \sup _{K_{j}}\left|\vec{p}_{j}\right|\right)^{k-1}\left(K\left(\beta_{1}, \ldots, \beta_{n}\right)\right)^{k}\right)
\end{aligned}
$$


and

$$
K\left(\beta_{1}, \ldots, \beta_{n}\right)=\frac{m_{2}^{\prime} \cdots m_{n}^{\prime}}{\rho^{\prime} \prod_{i=2}^{n} \inf _{V_{\beta_{1}}}\left(p_{i}^{0}\right)} \sum_{l \geq 0}\left(\Omega\left(\bar{x}_{2}, \ldots, \bar{x}_{n}, \bar{y}_{2}, \ldots, \bar{y}_{n}, \bar{z}\right)\right)^{l} .
$$

Take now $\left(q_{2}, \ldots, q_{n}\right) \in V_{\beta_{2}}^{2} \times \cdots \times V_{\beta_{n}}^{n}$; it results from (3.12), (3.13) and (3.14) that

$$
K\left(\beta_{1}, \ldots, \beta_{n}\right) \leq \frac{(1+\eta)^{n-1} m_{2}^{\prime} \cdots m_{n}^{\prime}}{\rho^{\prime} q_{2}^{0} \cdots q_{n}^{0}} \sum_{l \geq 0}\left((1+\eta)^{2} \tau_{L_{\beta_{1}}^{\prime}}\left(q_{2}, \ldots, q_{n}\right)\right)^{l} .
$$

So, if $\eta$ is chosen small enough

$$
K\left(\beta_{1}, \ldots, \beta_{n}\right) \leq \frac{(1+\eta)^{n-1} m_{2}^{\prime} \cdots m_{n}^{\prime}}{\rho^{\prime} q_{2}^{0} \cdots q_{n}^{0}}\left(1-(1+\eta)^{2} \tau_{L_{\beta_{1}}^{\prime}}\left(q_{2}, \ldots, q_{n}\right)\right)^{-1} .
$$

\section{Define}

$$
\begin{aligned}
R=\sup _{\left(L_{\beta}\right)_{\beta \in B}}\left(\sigma+2 \sum_{1<i<j}\left(\left(\sup _{K_{l}} p_{i}^{0}\right)\left(\sup _{K_{J}} p_{j}^{0}\right)\right.\right. \\
\\
\left.\left.\quad+\left(\sup _{K_{i}}\left|\vec{p}_{\imath}\right|\right)\left(\sup _{K_{J}}\left|\vec{p}_{J}\right|\right)+2 \sum_{j>1} \sup _{K_{J}}\left|\vec{p}_{j}\right|\right)\right) .
\end{aligned}
$$

We have

(3.16)

$$
\begin{aligned}
& C\left(k, \beta_{1}, \ldots, \beta_{n}\right) \\
& \leq(R \eta)^{k-1}\left((1+\eta)^{n-1} \frac{m_{2}^{\prime} \cdots m_{n}^{\prime}}{\rho^{\prime} q_{2}^{0} \cdots q_{n}^{0}}\right. \\
& \left.\quad \times\left(1-(1+\eta)^{2} \tau_{L_{\beta_{1}}^{\prime}}^{\prime}\left(q_{2}, \ldots, q_{n}\right)\right)^{-1}\right)^{k} .
\end{aligned}
$$

Choose $\eta^{\prime}>0$. If $\eta>0$ is small enough one has:

$$
\frac{R \eta(1+\eta)^{n-1} m_{2}^{\prime} \cdots m_{n}^{\prime}}{\rho^{\prime} q_{2}^{0} \cdots q_{n}^{0}}\left(1-(1+\eta)^{2} \tau_{L_{\beta_{1}}}^{\prime}\left(q_{2}, \ldots, q_{n}\right)\right)^{-1} \leq \eta^{\prime}
$$

and

$$
\begin{gathered}
\frac{(1+\eta)^{n-1} m_{2}^{\prime} \cdots m_{n}^{\prime}}{\rho^{\prime} q_{2}^{0} \cdots q_{n}^{0}}\left(1-(1+\eta)^{2} \tau_{L_{1}}^{\prime}\left(q_{2}, \ldots, q_{n}\right)\right)^{-1} \\
\leq\left(1+\eta^{\prime}\right) \nu_{L_{\beta_{1}}}^{\prime}\left(q_{2}, \ldots, q_{n}\right)
\end{gathered}
$$

independently of $\beta_{1}, \ldots, \beta_{n}$ and $\left(q_{2}, \ldots, q_{n}\right) \in V_{\beta_{2}}^{2} \times \cdots \times V_{\beta_{n}}^{n}$. 
From (3.5), (3.15), (3.17) and (3.18) one gets the inequality

$$
\begin{aligned}
& \left\|\mu \cdot \chi_{\beta_{1}}^{1} f_{1} \otimes \cdots \otimes \chi_{\beta_{n}}^{n} f_{n}\right\| \\
& \quad \leq\left(1+\eta^{\prime}\right) \nu_{L_{\beta_{1}}^{\prime}}^{\prime}\left(q_{2}, \ldots, q_{n}\right)\left(1+\frac{\eta^{\prime}}{1-\eta^{\prime}}\right)\left\|\chi_{\beta_{1}}^{1} f_{1}\right\| \cdots\left\|\chi_{\beta_{n}}^{n} f_{n}\right\| .
\end{aligned}
$$

Since $\nu_{L_{1}}^{\prime}\left(q_{2}, \ldots, q_{n}\right) \leq \rho^{-1}$, we finally have

$$
\begin{aligned}
& \left\|\mu \cdot \chi_{\beta_{1}}^{1} f_{1} \otimes \cdots \otimes \chi_{\beta_{n}}^{n} f_{n}\right\| \\
& \quad \leq\left(1+\eta^{\prime}\right) \rho^{-1}\left(1+\frac{\eta^{\prime}}{1-\eta^{\prime}}\right)\left\|\chi_{\beta_{1}}^{1} f_{1}\right\| \cdots\left\|\chi_{\beta_{n}}^{n} f_{n}\right\| .
\end{aligned}
$$

Since the functions $\left\{\chi_{\beta_{1}}^{i} f\right\}_{\beta_{i} \in B}$ are orthogonal for each $i \in\{1, \ldots, n\}$ and since $f_{i}=\Sigma_{\beta_{i} \in B} \chi_{\beta_{i}}^{i} f$, we have

$$
\left\|\mu \cdot f_{1} \otimes \cdots \otimes f_{n}\right\| \leq\left(1+\eta^{\prime}\right) \rho^{-1}\left(1+\frac{\eta^{\prime}}{1-\eta^{\prime}}\right)\left\|f_{1}\right\| \cdots\left\|f_{n}\right\| .
$$

Since $\eta^{\prime}$ is arbitrarily small

$$
\left\|\mu \cdot f_{1} \otimes \cdots \otimes f_{n}\right\| \leq \rho^{-1}\left\|f_{1}\right\| \cdots\left\|f_{n}\right\|
$$

which proves that $R_{\lambda}$ maps $Y$ to itself and that its norm, as an operator on $Y$, is smaller than $\rho^{-1}$.

The proof that $Q^{\prime}$ is closable in $Y$ and that $R_{\lambda}$ is its resolvent is the same as in part (a). This completes the proof of Proposition 3.1.

Proposition 3.3. Suppose that $m_{1} \neq 0, \ldots, m_{n} \neq 0$ and that $\lambda<$ $\left(m_{1}+\cdots+m_{n}\right)^{2}$. The operator $Q\left(m_{1}, \ldots, m_{n}, \varepsilon\right)$ is closable in $\mathfrak{X}$ and has $a$ resolvent $R_{\lambda}=\left(\vec{Q}\left(m_{1}, \ldots, m_{n}, \varepsilon\right)-\lambda\right)^{-1}$. Moreover

$$
\left\|R_{\lambda}\right\| \leq\left(\left(m_{1}+\cdots+m_{n}\right)^{2}-\lambda\right)^{-1} .
$$

Proof. The norm of the operator $R_{\lambda}$ defined in Proposition 3.1 on $E\left(K_{1}\right) \hat{\otimes}_{\pi} \cdots \hat{\otimes}_{\pi} E\left(K_{n}\right)$ satisfies inequality (3.19). Functions with compact support form a dense set in $E\left(M\left(m_{i}, \varepsilon\right)\right)$. Therefore $R_{\lambda}$ has a unique extension (denoted again by $R_{\lambda}$ ) to $\mathfrak{X}$, as a continuous linear mapping, satisfying (3.19). One then proves as in part (a) of Proposition 3.1 that $Q$ is closable in $\mathfrak{X}$ and that $R_{\lambda}=(\bar{Q}-\lambda)^{-1}$.

The Fréchet space $E_{\tau}(M(m, \varepsilon))$ is the set of functions $f$ from $M(m, \varepsilon)$ to $\Sigma$ such that the function $p \rightarrow\left|p^{\mu}\right|^{n} f(p), \mu=0,1,2,3, n \in \mathbf{N}$, belongs to $L^{q}(M(m, \varepsilon), \Sigma)$.

On $D_{Q}$ the operators $Q$ and $P_{i}^{\mu}$ (defined by $P_{i}^{\mu} f\left(p_{1}, \ldots, p_{n}\right)=$ $\left.p_{i}^{\mu} f\left(p_{1}, \ldots, p_{n}\right), i=1, \ldots, n\right)$ commute. 
Therefore, from the definition of the topology on $E_{\tau}\left(M\left(m_{1}, \varepsilon\right)\right) \hat{\otimes}_{\pi}$ $\cdots \hat{\otimes}_{\pi} E_{\tau}\left(M\left(m_{n}, \varepsilon\right)\right), Q$ has a unique continuous extension (denoted again by $Q$ ) to this space and, using Proposition 3.3, we have:

Proposition 3.4. Suppose that $m_{1} \neq 0, \ldots, m_{n} \neq 0$ and that $\lambda<$ $\left(m_{1}+\cdots+m_{n}\right)^{2}$. On $E_{\tau}\left(M\left(m_{1}, \varepsilon\right)\right) \hat{\otimes}_{\pi} \cdots \hat{\otimes}_{\pi} E_{\tau}\left(M\left(m_{n}, \varepsilon\right)\right)$ the operator $Q\left(m_{1}, \ldots, m_{n}, \varepsilon\right)-\lambda$ has a continuous inverse

$$
R_{\lambda}=\left(Q\left(m_{1}, \ldots, m_{n}, \varepsilon\right)-\lambda\right)^{-1}
$$

4. Formal properties of some non linear representations. Suppose given integers $n_{1} \geq 0, n_{2} \geq 0, n_{3} \geq 0$ and $n_{4} \geq 0$; put $n=n_{1}+n_{2}+$ $n_{3}+n_{4}$. Suppose also given a continuous linear representation $(U, E)$ of the Poincaré group, $U=\bigoplus_{i=1}^{n} U^{m_{l}, \varepsilon_{l}}$ on $E=\bigoplus_{i=1}^{n} E\left(M\left(m_{i}, \varepsilon_{l}\right)\right)$ such that

1. $m_{i}>0$ and $\varepsilon_{i}=-1$ if $i=1, \ldots, n_{1}$,

2. $m_{i}>0$ and $\varepsilon_{i}=+1$ if $i=n_{1}+1, \ldots, n_{1}+n_{2}$,

3. $m_{l}=0$ and $\varepsilon_{i}=-1$ if $i=n_{1}+n_{2}+1, \ldots, n_{1}+n_{2}+n_{3}$,

4. $m_{i}=0$ and $\varepsilon_{i}=+1$ if $i=n_{1}+n_{2}+n_{3}+1, \ldots, n$.

Denote by $E^{-}$(resp. $\left.E^{+}\right)$the space $\bigoplus_{i=1}^{n_{1}} E\left(m_{l}, \varepsilon_{l}\right)$ (resp. $\left.\bigoplus_{l=n_{1}+1}^{n_{1}+n_{2}} E\left(m_{i}, \varepsilon_{i}\right)\right)$, and by $P^{-}$(resp. $P^{+}$, resp. $\left.P^{m_{i}, \varepsilon_{i}}\right)$ the projector on $E^{-}$(resp. $E^{+}$, resp. $\left.E\left(M\left(m_{i}, \varepsilon_{i}\right)\right)\right)$. One labels with a subscript the corresponding projectors $\left(P_{\tau}^{-}, P_{\tau}^{+}\right.$, and $\left.P_{\tau}^{m_{l}, \varepsilon_{t}}\right)$ restricted to $E_{\tau}$.

Define $E_{c}=\bigoplus_{i=1}^{n} E_{c}\left(M\left(m_{l}, \varepsilon_{i}\right)\right)$. As a topological vector space, $E_{c}\left(M\left(m_{i}, \varepsilon_{l}\right)\right)=\lim$ ind $E(K)$, where $K \subset M\left(m_{i}, \varepsilon_{i}\right)$ is compact.

Given a topological vector space $Y$, denote by $\mathrm{t}\left(E_{\tau}, Y\right)$ the set of power series $f \in \mathfrak{F}\left(E_{\tau}, Y\right)$ of the form $f=\Sigma_{k \geq 2} f^{k}, f^{k} \in \mathfrak{R}_{k}\left(E_{\tau}, Y\right)$, such that $f^{k}\left(P_{\tau}^{m_{t_{1}}, \varepsilon_{11}} \otimes \cdots \times P_{\tau}^{m_{i_{k}}, \varepsilon_{t_{k}}}\right)=0$ whenever at least one of the following conditions is satisfied

$\left(\mathrm{C}_{1}\right) \varepsilon_{i_{1}}, \ldots, \varepsilon_{i_{k}}$ are not all equal, or

$\left(\mathrm{C}_{2}\right)$ there exists $j \in\left\{i_{1}, \ldots, i_{k}\right\}$ such that $m_{j}=0$.

For short one writes $\mathfrak{R}_{n}(X)=\mathfrak{R}_{n}(X, X), \mathfrak{F}(X)=\mathfrak{F}(X, X)$ ( $X$ being a topological vector space).

Proposition 4.1. Suppose that $G$ is either $P$ or $T_{4}$ and that $(S, E)$ is a smooth representation of $G$ in $E$ (for this notion see [1, Definition 6]), $S_{g}=\Sigma_{n \geq 1} S_{g}^{n}, S_{g}^{n} \in \mathfrak{R}_{n}(E)$, such that

(1) $S^{1}=\left.U\right|_{G}$.

(2) For any $i, j$ such that $m_{i} m_{j} \neq 0$, then $m_{i}+m_{j}>m_{k}$ for any $k$; $i, j, k \in\{1, \ldots, n\}$.

Then, there exists a unique $A \in \mathrm{t}\left(E_{\tau}, E_{\tau}\right)$ such that:

$$
\left((I+A) S_{g}^{1}-S_{g}(I+A)\right) P_{\tau}^{ \pm}=0 .
$$


Proof. One denotes by $E_{\infty}$ the space of differentiable vectors of $\left.U\right|_{G}$. $(S, E)$ being smooth, the mapping $g \rightarrow S_{g^{-1}}^{1} S_{g}^{n}$ is $C^{\infty}$ from $G$ to $\mathfrak{R}_{n}(E)$ and $S_{g}^{n} \in \mathfrak{R}_{n}\left(E_{\infty}\right)$ for any $g \in G$. Since $T_{4}$ is an invariant subgroup of $G$, and $S_{x}^{1} S_{g}^{1} \varphi=S_{g}^{1} S_{g^{-1} x g}^{1} \varphi, x \in T_{4}, g \in G$, the mapping $x \rightarrow S_{x}^{1} S_{g}^{1} \varphi$ is $C^{\infty}$ from $T_{4}$ to $E$ whenever $\varphi \in E_{\tau}$. Therefore, $S_{g}^{1} \in \mathfrak{R}_{1}\left(E_{\tau}\right)$ for any $g \in G$. Using the relation $S_{x} S_{g} \varphi=S_{g} S_{g^{-1} x g} \varphi$ one sees by induction that $S_{g}^{n} \in \mathfrak{L}_{n}\left(E_{\tau}\right)$ for any $g \in G$. Take $l \geq 2$ and suppose that there exists a unique polynomial $A_{l-1}=A^{2}+\cdots+A^{l-1} \in \mathrm{t}\left(E_{\tau}, E_{\tau}\right)$ such that

$$
\left(\left(I+A_{l-1}\right) S_{g}^{1}-S_{g}\left(1+A_{l-1}\right)\right)^{k} P_{\tau}^{ \pm}=0
$$

for $1 \leq k \leq l-1$. Then,

$$
\begin{aligned}
\left(S_{g g^{\prime}}\left(I+A_{l-1}\right)\right)^{l} P_{\tau}^{ \pm}= & \left(S_{g} S_{g^{\prime}}\left(I+A_{l-1}\right)\right)^{l} P_{\tau}^{ \pm}=S_{g}^{1}\left(S_{g^{\prime}}\left(I+A_{l-1}\right)\right)^{l} P_{\tau}^{ \pm} \\
& +\left(\left(\sum_{n \geq 2} S_{g}^{n}\right) S_{g^{\prime}}\left(I+A_{l-1}\right)\right)^{l} P_{\tau}^{ \pm}
\end{aligned}
$$

Now, from (4.2), one has

$$
\left(\left(\sum_{n \geq 2} S_{g}^{n}\right) S_{g^{\prime}}\left(I+A_{l-1}\right)\right)^{l} P_{\tau}^{ \pm}=\left(\left(\sum_{n \geq 2} S_{g}^{n}\right)\left(I+A_{l-1}\right) S_{g^{\prime}}^{1}\right)^{l} P_{\tau}^{ \pm} .
$$

Consequently, defining $R_{g}^{ \pm}=S_{g}^{1}\left(\sum_{n \geq 2} S_{g^{-1}}^{n}\left(1+A_{l-1}\right)\right)^{l} P_{\tau}^{ \pm}$, we have

$$
R_{g g^{\prime}}^{ \pm}=R_{g}^{ \pm}+S_{g}^{1} R_{g^{\prime}}^{ \pm} S_{g^{-1}}^{1} \text {. }
$$

This means that $R^{ \pm}$is a 1 -cocycle on $G$ with coefficients in $\Omega_{l}\left(E_{\tau}\right)$, the action of $G$ being defined by $g \rightarrow S_{g}^{1} Z S_{g^{-1}}^{1}, Z \in \mathfrak{Q}_{l}\left(E_{\tau}\right)$.

Consider now the cocycle $d R^{ \pm}$, on the Lie algebra $t_{4}$ of $T_{4}$, defined by

$$
d R_{X}^{ \pm} \varphi=\frac{d}{d s}\left(R_{\exp s X}^{ \pm} \varphi\right)_{s=0}, \quad X \in \mathfrak{t}_{4}, \varphi \in E_{\tau} .
$$

Then $d R_{X}^{ \pm} \in \mathfrak{R}_{l}\left(E_{\tau}\right)$. By [8, Lemma 6.3], $d R_{X}^{ \pm}$has a linear extension (again denoted by $d R^{ \pm}$) to the universal enveloping algebra $\mathfrak{U}\left(\mathrm{t}_{4}\right)$ of $\mathrm{t}_{4}$ such that

$$
d R_{X Y}^{ \pm}=d S_{X}^{1} d R_{Y}^{ \pm}+d R_{X}^{ \pm} * d S_{Y}^{1}, \quad X, Y \in \mathfrak{U}\left(\mathrm{t}_{4}\right)
$$

(because $d R_{Y}^{ \pm} * d S_{X}^{1}=d R_{Y}^{ \pm} d\left(\hat{\otimes}_{\pi}^{l} S^{1}\right)_{X}$ ).

Given a Lorentz basis $\left\{X_{0}, X_{1}, X_{2}, X_{3}\right\}$ in $\mathrm{t}_{4}$, we have the central element $Q=\Sigma_{\mu=0}^{3} \eta_{\mu} X_{\mu}^{2}$, where $\eta_{0}=-1, \eta_{1}=\eta_{2}=\eta_{3}=1$. Therefore

$$
d R_{Q}^{ \pm}=\sum_{\mu=0}^{3} \eta_{\mu}\left(d S_{X_{\mu}}^{1} d R_{X_{\mu}}^{ \pm}+d R_{X_{\mu}}^{ \pm} * d S_{X_{\mu}}^{1}\right) \text {. }
$$


If $X \in \mathrm{t}_{4}$, we have

$$
\begin{gathered}
d R_{X Q}^{ \pm}=d S_{X}^{1} d R_{Q}^{ \pm}+d R_{X}^{ \pm} d\left(\otimes^{\prime} S^{1}\right)_{Q}, \\
d R_{Q X}^{ \pm}=d S_{Q}^{1} d R_{X}^{ \pm}+d R_{Q}^{ \pm} * d S_{X}^{1} .
\end{gathered}
$$

Equalities $X Q=Q X,(4.4),(4.5)$ and $P_{\tau}^{m_{J}, \varepsilon_{J}} d S_{Q}^{1}=m_{j}^{2} P_{\tau}^{m_{J}, \varepsilon_{J}}$ imply (for $\varepsilon_{i_{1}}=\cdots=\varepsilon_{i_{1}}=\varepsilon= \pm 1, R^{\varepsilon}$ meaning $R^{+}$or $R^{-}$according to the sign of E) that

$$
\begin{array}{r}
P^{m_{j}, \varepsilon_{j}}\left(d R_{X}^{\varepsilon}\left(m_{j}^{2}-Q\left(m_{i_{1}}, \ldots, m_{i_{l}}, \varepsilon\right)\right)-\left(d S_{X}^{1} d R_{Q}^{\varepsilon}-d R_{Q}^{\varepsilon} * d S_{X}^{1}\right)\right) \\
\times\left(P_{\tau}^{m_{i_{1}}, \varepsilon_{i_{1}}} \otimes \cdots \otimes P_{\tau}^{m_{i,}, \varepsilon_{\ell_{l}}}\right)=0 .
\end{array}
$$

When $m_{i_{1}} \neq 0, \ldots, m_{i_{l}} \neq 0$ and $\varepsilon_{i_{1}}=\cdots=\varepsilon_{i_{k}}=\varepsilon= \pm 1$, we shall define

$$
\begin{aligned}
& A^{l}\left(P_{\tau}^{m_{i_{1}}, \varepsilon_{i_{1}}} \otimes \cdots \otimes P_{\tau}^{m_{l_{l}}, \varepsilon_{t_{l}}}\right) \\
& \quad=\sum_{j=1}^{n} P^{m_{\jmath}, \varepsilon_{j}} d R_{Q}^{\varepsilon}\left(m_{j}^{2}-\bar{Q}\left(m_{i_{1}}, \ldots, m_{i_{l}}, \varepsilon\right)\right)^{-1} P_{\tau}^{m_{l_{1}}, \varepsilon_{l_{1}}} \otimes \cdots \otimes P_{\tau}^{m_{i}, \varepsilon_{l_{l}}}
\end{aligned}
$$

and take $A^{l}\left(P_{\tau}^{m_{i_{1}}, \varepsilon_{i_{1}}} \otimes \cdots \otimes P_{\tau}^{m_{t r} \varepsilon_{i_{1}}}\right)=0$ if $\varepsilon_{i_{1}}, \ldots, \varepsilon_{i_{l}}$ are not all equal or if there exists $j \in\left\{i_{1}, \ldots, i_{l}\right\}$ such that $m_{j}=0$. We have

$$
d R_{X}^{\varepsilon}=d S_{X}^{1} A^{l} P_{\tau}^{\varepsilon}-\left(A^{l} P_{\tau}^{\varepsilon}\right) * d S_{X}^{1}, \quad x \in \mathfrak{t}_{4},
$$

which means that $d R^{\varepsilon}$ is the coboundary of $A^{l} P_{\tau}^{\varepsilon}$ on $t_{4}$. Consequently

$$
R_{x}^{\varepsilon}=S_{x}^{1}\left(A^{l} P_{\tau}^{\varepsilon}\right) S_{x^{-1}}^{1}-A^{l} P_{\tau}^{\varepsilon}, \quad x \in T_{4} .
$$

Define $\tilde{R}_{g}^{ \pm}=R_{g}^{ \pm}-S_{g}^{1}\left(A P_{\tau}^{ \pm}\right) S_{g^{-1}}^{1}-A^{l} P_{\tau}^{ \pm}, g \in G$. We have $\tilde{R}_{x}^{ \pm}=0$ for $x \in T_{4} . R^{ \pm}$is a 1 -cocycle on $G$ with coefficients in $\mathfrak{L}_{l}\left(E_{\tau}\right)$. Writing $\tilde{R}_{g^{-1} x g}^{ \pm}=0, x \in T_{4}$, one gets

$$
S_{x}^{1} \tilde{R}_{g}^{ \pm} S_{x^{-1}}^{1}=-S_{g}^{1} \tilde{R}_{g^{-1}}^{ \pm} S_{g^{-1}}^{1}, \quad x \in T_{4}, g \in G .
$$

The right-hand side of (4.9) being independent of $X$ one obtains

$$
d S_{X}^{1} \tilde{R}_{g}^{ \pm}-\tilde{R}_{g}^{ \pm} * d S_{X}^{1}=0, \quad X \in \mathrm{t}_{4} .
$$

Therefore

$$
\sum_{\mu=0}^{3} \eta_{\mu}\left(\left(d S_{X_{\mu}}^{1}\right)^{2} \tilde{R}_{g}^{ \pm}-\left(\left(\tilde{R}_{g}^{ \pm} * d S_{X_{\mu}}^{1}\right) * d S_{X_{\mu}}^{1}\right)\right)=0
$$

which implies that

$$
P^{m_{j}, \varepsilon_{j}} \tilde{R}_{g}^{ \pm}\left(m_{j}^{2}-Q\left(m_{i_{1}}, \ldots, m_{i_{l}}, \varepsilon_{i_{1}}\right)\right)\left(P_{\tau}^{m_{t_{1}}, \varepsilon_{t_{1}}} \otimes \cdots \otimes P_{\tau}^{m_{i l}, \varepsilon_{t_{i}}}\right)=0 .
$$


Proposition 3.4 implies then that $\tilde{R}_{g}^{ \pm}=0$. Going back to the definition of $R_{g}^{ \pm}$, this means that

$$
S_{g}^{1}\left(\left(\sum_{n \geq 2} S_{g^{-1}}^{n}\right)\left(I+A_{l-1}\right)\right)^{l} P_{\tau}^{ \pm}=S_{g}^{1} A^{l} P_{\tau}^{ \pm} S_{g^{-1}}^{1}-A^{l} P_{\tau}^{ \pm},
$$

which can be rewritten with $A_{l}=A_{l-1}+A^{l}$

$$
\left(I+A_{l}\right)^{l} S_{g}^{1} P_{\tau}^{ \pm}=\left(S_{g}\left(I+A_{l}\right)\right)^{l} P_{\tau}^{ \pm} .
$$

One then defines $A=\Sigma_{l \geq 2} A^{l}$. There remains to prove the uniqueness of $A^{l} \in \mathrm{t}\left(E_{\tau}, E_{\tau}\right)$. Suppose that there exists a second one, $\tilde{A}^{l}$. Equality (4.10) implies that

$$
S_{x}^{1}\left(A^{l}-\tilde{A}^{l}\right)-\left(A^{l}-\tilde{A}^{l}\right) S_{x}^{1}=0, \quad x \in T_{4} .
$$

Therefore

$$
d S_{X}^{1}\left(A^{l}-\tilde{A}^{l}\right)-\left(A^{l}-\tilde{A}^{l}\right) * d S_{X}^{1}=0, \quad X \in \mathrm{t}_{4} .
$$

The same calculation as above proves then that $A^{l}=\tilde{A}^{l}$.

REMARK 4.2. Consider the series $A \in \mathrm{t}\left(E_{\tau}, E_{\tau}\right)$ satisfying the conclusions of Proposition 4.1. The formal power series $I+A$ has an inverse in $\mathfrak{F}\left(E_{\tau}\right)$. Consider, on $E_{\tau}$, the formal representation (for this notion see [1, Definition 1]) $\tilde{S}_{g}=(I+A)^{-1} S_{g}(I+A)$. It satisfies $\left(S_{g}^{1}-\tilde{S}_{g}\right) P_{\tau}^{ \pm}=0$.

5. Convergence of the intertwining power series. Given two topological vector spaces $X$ and $Y$, one denotes by $\tilde{\mathfrak{F}}(X, Y)$ the subspace of $\mathfrak{F}(X, Y)$ of the series $f=\Sigma_{n \geq 2} f^{n}, f^{n} \in \mathfrak{R}_{n}(X, Y)$. If $X$ and $Y$ are Banach spaces and $r>0$ one defines $\tilde{\mathfrak{S}}_{r}(X, Y)=\mathscr{S}_{r}(X, Y) \cap \tilde{\mathfrak{F}}(X, Y)$. We shall write $\tilde{\mathfrak{F}}(E)=\tilde{\mathfrak{F}}(E, E), \mathfrak{S}_{r}(E)=\mathfrak{S}_{r}(E, E)$ and $\tilde{\mathfrak{S}}_{r}(E)=\tilde{\mathscr{E}}_{r}(E, E)$, and keep the hypotheses of part 4.

A linear operator $W$ in $\tilde{\mathfrak{F}}\left(E_{\tau}, E\right)$ can be defined by:

(1) $W\left(f^{k}\left(P_{\tau}^{m_{t,}, \varepsilon_{t_{1}}} \otimes \cdots \otimes P_{\tau}^{m_{t_{k}}, \varepsilon_{t k}}\right)\right)=0$ if condition $\left(\mathrm{C}_{1}\right.$ or $\left.\mathrm{C}_{2}\right)$ of part 4 is satisfied.

(2) If condition $\left(\mathrm{C}_{1}\right.$ or $\left.\mathrm{C}_{2}\right)$ is not satisfied:

$$
\begin{aligned}
W\left(f^{k}\left(P_{\tau}^{m_{i_{1}}, \varepsilon_{i_{1}}} \otimes \cdots \otimes P_{\tau}^{m_{t_{k}}, \varepsilon_{t_{k}}}\right)\right) & \\
=\sum_{j=1}^{n} P^{m_{J}, \varepsilon_{j}} f^{k}\left(\left(m_{j}^{2}-\bar{Q}\left(m_{i_{1}} \cdots m_{i_{k}}, \varepsilon_{i_{1}}\right)\right)^{-1}\right. & \\
& \left.\quad \times P_{\tau}^{m_{t_{1}}, \varepsilon_{i_{1}}} \otimes \cdots \otimes P_{\tau}^{m_{t_{k}}, \varepsilon_{t_{k}}}\right) .
\end{aligned}
$$


Obviously $W(f) \in \mathrm{t}\left(E_{\tau}, E\right)$. Moreover, it results from Proposition 3.3 that there exists a constant $C$ such that

$$
\left\|\left(m_{j}^{2}-\bar{Q}\left(m_{i_{1}}, \ldots, m_{i_{k}}, \varepsilon_{i_{1}}\right)\right)^{-1}\right\| \leq C k^{-2} .
$$

Consequently, given compact sets $K_{i} \subset M\left(m_{i}, \varepsilon_{i}\right), i=1, \ldots, n, W$ is continuous from $\tilde{\mathfrak{F}}_{r}\left(\bigoplus_{i=1}^{n} E\left(K_{i}\right), E\right)$ to itself.

Lemma 5.1. Suppose that $G$ is either $P$ or $T_{4}$ and that $(S, E)$ is a smooth representation of $G$ in $E$ satisfying the hypotheses of Proposition 4.1. Given $K_{i}$ compact in $M\left(m_{i}, \varepsilon_{i}\right)(i=1, \ldots, n)$ there exists $r>0$ such that the series $A \in \mathfrak{t}\left(E_{\tau}, E_{\tau}\right)$ defined by Proposition 4.1 belongs to $\tilde{\mathfrak{F}}_{r}\left(\bigoplus_{i=1}^{n} E\left(K_{i}\right), E\right)$.

Proof. We shall write equality 4.1 in a form which will be more convenient to prove the convergence of $A$.

Take $x \in \mathrm{t}_{4}$; equality (4.1) implies, by differentiation, that

$$
\left((I+A) * d S_{X}^{1}-d S_{X}(I+A)\right) P_{\tau}^{ \pm}=0 .
$$

Let $T_{X}=\Sigma_{n \geq 2} d S_{X}^{n}$. Equality (5.2) now writes

$$
d S_{X}^{1}\left(A P_{\tau}^{ \pm}\right)-\left(A P_{\tau}^{ \pm}\right) * d S_{X}^{1}=-T_{X}(I+A) P_{\tau}^{ \pm}
$$

which in turn implies that

$$
\begin{aligned}
d S_{X}^{1} d S_{X}^{1}\left(A P_{\tau}^{ \pm}\right)- & \left(\left(A P_{\tau}^{ \pm}\right) * d S_{X}^{1}\right) * d S_{X}^{1} \\
& =-\left(d S_{X}^{1} T_{X}(I+A)+\left(T_{X}(I+A)\right) * d S_{X}^{1}\right) P_{\tau}^{ \pm} .
\end{aligned}
$$

Therefore, if $\varepsilon_{i_{1}}=\cdots=\varepsilon_{i_{k}}= \pm 1$ and $m_{i_{1}} \neq 0, \ldots, m_{i_{k}} \neq 0$, we have

$$
\begin{aligned}
P^{m_{j}, \varepsilon_{j}} A^{k}\left(m_{j}^{2}-Q\left(m_{i_{1}}, \ldots, m_{i_{k}}, \varepsilon_{i_{1}}\right)\right) P_{\tau}^{m_{\imath_{1}}, \varepsilon_{i_{1}}} \otimes \cdots \otimes P_{\tau}^{m_{\iota_{k}}, \varepsilon_{i_{k}}} & -P^{m_{\jmath}, \varepsilon_{j}}\left[\sum_{\mu=0}^{3} \eta_{\mu}\left(d S_{X_{\mu}}^{1} T_{X_{\mu}}(I+A)+\left(T_{X_{\mu}}(I+A)\right) * d S_{X_{\mu}}^{1}\right)\right] \\
& \quad \times P_{\tau}^{m_{i_{1}}, \varepsilon_{i_{1}}} \otimes \cdots \otimes P_{\tau}^{m_{\imath_{k}}, \varepsilon_{l_{k}}}
\end{aligned}
$$

which is equivalent to

(5.4) $A=-W\left(\sum_{\mu=0}^{3} \eta_{\mu}\left(d S_{X_{\mu}}^{1} T_{X_{\mu}}(I+A)+\left(T_{X_{\mu}}(I+A)\right) * d S_{X_{\mu}}^{1}\right)\right)$.

Now, using the fact that $A$ satisfies equality (5.3) one has

$$
\begin{aligned}
d S_{X_{\mu}}^{1} T_{X_{\mu}}(I+A) P_{\tau}^{ \pm}=\left(\left[d S_{X_{\mu}}^{1}, T_{X_{\mu}}\right] *(I+A)\right. & -\left(T_{X_{\mu}} * T_{X_{\mu}}\right)(I+A) \\
& \left.+\left(T_{X_{\mu}}(1+A)\right) * d S_{X_{\mu}}^{1}\right) P_{\tau}^{ \pm} .
\end{aligned}
$$


Consequently, since $A \in \mathrm{t}\left(E_{\tau}, E_{\tau}\right)$, one gets (from (5.4)) the equation:

$$
A=N(A)
$$

with

$$
\text { (5.6) } \begin{array}{r}
N(A)=-W\left(\sum _ { \mu = 0 } ^ { 3 } \eta _ { \mu } \left(\left[d S_{X_{\mu}}^{1}, T_{X_{\mu}}\right] *(I+A)-\left(T_{X_{\mu}} * T_{X_{\mu}}\right)(I+A)\right.\right. \\
\left.\left.+2\left(T_{X_{\mu}}(I+A)\right) * d S_{X_{\mu}}^{1}\right)\right) .
\end{array}
$$

Notice that the term of degree $k$ in $N(A)$ depends only on $A^{2}, \ldots, A^{k-1}$. Therefore equation (5.5) has one and only one solution in $\tilde{\mathfrak{F}}\left(E_{c}, E\right)$. Since, as was just proved, the solution of (4.1) is solution of (5.5), it is sufficient to solve (5.5) in $\tilde{\mathfrak{F}}_{r}\left(\bigoplus_{i=1}^{n} E\left(K_{i}\right), E\right)$ for some $r>0$.

$(S, E)$ being a smooth representation, there exists $\lambda_{1}>0$ such that $\left(g, g^{\prime}\right) \rightarrow S_{g^{\prime} g^{-1}}^{1} S_{g g^{\prime-1}}$ is $C^{\infty}$ from a neighbourhood of the identity in $G \times G$ to $\mathfrak{S}_{\lambda_{1}}(E)$. Writing $g=\exp s X$ and $g^{\prime}=\exp s^{\prime} X, X \in \mathrm{t}_{4}$, one gets

$$
\frac{\partial^{2}}{\partial s \partial s^{\prime}}\left(S_{g^{\prime} g^{-1}}^{1} S_{g g^{\prime-1}}\right)_{s=s^{\prime}=0}=d S_{X}^{1} T_{X} \varphi-T_{X} * d S_{X} \varphi \quad \text { with } \varphi \in E_{\infty} .
$$

Therefore

$$
\left[d S_{X}^{1}, T_{X}\right]_{*} \varphi=\frac{\partial^{2}}{\partial s \partial s^{\prime}}\left(S_{g^{\prime} g^{-1}}^{1} S_{g g^{\prime-1}}\right)_{s=s^{\prime}=0} \varphi+T_{X} * T_{X} \varphi .
$$

Thus $\left[d S_{X}^{1}, T_{X}\right]_{*} \in \mathfrak{S}_{\lambda_{2}}(E)$ for some $\lambda_{2}>0$. Take now

$$
\begin{gathered}
f=\sum_{k \geq 2} f^{k} \in \mathfrak{S}_{\lambda}(E), \quad h_{1}=\sum_{k \geq 2} h_{1}^{k} \text { and } \\
h_{2}=\sum_{k \geq 2} h_{2}^{k} \text { in } \mathfrak{S}_{\lambda^{\prime}}\left(\bigoplus_{i=1}^{n} E\left(K_{i}\right), E\right)
\end{gathered}
$$

with $0<4 \lambda^{\prime}<\lambda$. We have

$$
\left\|f\left(I+h_{1}\right)\right\|_{\lambda^{\prime}} \leq \sum_{k \geq 2}\left\|f^{k}\right\|\left(\lambda^{\prime}+\left\|h_{1}\right\|_{\lambda^{\prime}}\right)^{k}
$$

and

$$
f\left(I+h_{2}\right)-f\left(I+h_{1}\right)=\int_{0}^{1} \frac{d}{d s} f(a(s)) d s
$$

with $a(s)=I+h_{1}+s\left(h_{2}-h_{1}\right)$. Since

$$
\frac{d}{d s} f(a(s))=\sum_{k \geq 2} k f^{k}\left(a(s), \ldots, a(s), h_{2}-h_{1}\right),
$$


we have

$$
\begin{aligned}
\left\|\frac{d}{d s} f(a(s))\right\|_{\lambda^{\prime}} \leq & \sum_{k \geq 1} \lambda^{\prime k} \sum_{2 \leq p \leq k} p\left\|f^{p}\right\| \\
& \times\left(\sum_{i_{1}+\cdots+i_{p}=k}\left\|a^{i_{1}}(s)\right\| \cdots\left\|a^{i_{p-1}}(s)\right\|\left\|h_{2}^{i_{1}}-h_{1_{p}}^{i_{p}}\right\|\right) \\
\leq & \left(\sum_{p \geq 2} p\|a(s)\| p_{\lambda^{\prime}}^{-1}\left\|f^{p}\right\|\right)\left\|h_{2}-h_{1}\right\|_{\lambda^{\prime}} .
\end{aligned}
$$

Now, if $\left\|h_{1}\right\|_{\lambda^{\prime}} \leq \lambda^{\prime}$ and $\left\|h_{2}\right\|_{\lambda^{\prime}} \leq \lambda^{\prime}$ then $\|a(s)\|_{\lambda^{\prime}} \leq 4 \lambda^{\prime}$, and there exists a constant $C\left(\lambda^{\prime}\right)$ such that $\lim _{\lambda^{\prime} \rightarrow 0} C\left(\lambda^{\prime}\right)=0$ and

$$
\left\|f\left(I+h_{2}\right)-f\left(I+h_{1}\right)\right\|_{\lambda^{\prime}} \leq C\left(\lambda^{\prime}\right)\left\|h_{2}-h_{1}\right\|_{\lambda^{\prime}} .
$$

It results from (5.7), (5.8), and from the fact that $W$ is continuous from $\tilde{\mathfrak{S}}_{\lambda}\left(\oplus_{i=1}^{n} E\left(K_{i}\right), E\right)$ to itself for any $\lambda>0$, that for $r>0$ small enough the mapping

$$
\begin{aligned}
A & \rightarrow f_{1}(I+A) \\
& =-W\left(\sum_{\mu=0}^{3} \eta_{\mu}\left(\left[d S_{X_{\mu}}^{1}, T_{X_{\mu}}\right]_{*}(I+A)\right)-\left(T_{X_{\mu}} * T_{X_{\mu}}\right)(I+A)\right)
\end{aligned}
$$

maps the closed ball of radius $r$ to the ball of radius $r / 2$ in $\tilde{\mathfrak{S}}_{r}\left(\bigoplus_{i=1}^{n} E\left(K_{i}\right), E\right)$ and

$$
\left\|f_{1}\left(I+A_{1}\right)-f_{1}\left(I+A_{2}\right)\right\|_{r} \leq \frac{\delta}{2}\left\|A_{1}-A_{2}\right\|_{r},
$$

with $\delta<1$, and $A_{1}, A_{2}$ in the closed ball of radius $r$.

Now, using the fact that $d S_{X_{u}}^{1}$ is bounded on $E\left(K_{i}\right)$, from inequality (5.1) and again from (5.7) and (5.8) one sees that, for $r>0$ small enough, the mapping

$$
A \rightarrow f_{2}(I+A)=-2 W\left(\sum_{\mu=0}^{3}\left(T_{X_{\mu}}(1+A)\right) * d S_{X_{\mu}}^{1}\right)
$$

maps the closed ball of radius $r$ to the ball of radius $r / 2$ in $\tilde{\mathfrak{S}}_{r}\left(\bigoplus_{i=1}^{n} E\left(K_{i}\right), E\right)$ and,

$$
\left\|f_{2}\left(I+A_{1}\right)-f_{2}\left(I+A_{2}\right)\right\|_{r} \leq \frac{\delta}{2}\left\|A_{1}-A_{2}\right\|_{r},
$$

for $A_{1}, A_{2}$ in the closed ball of radius $r$. 
REMARK 5.2. This was the point where inequality (5.1) is important. In fact inequalities of the type $\left\|m_{j}^{2}-\bar{Q}\left(m_{i_{1}}, \ldots, m_{i_{k}}, \varepsilon_{i_{1}}\right)\right\|^{-1} \leq C k^{-1}$ would be sufficient to be still in the situation of no "small denominators". Going back to the proof of Proposition 5.1, the mapping

$$
A \rightarrow N(A)=f_{1}(I+A)+f_{2}(I+A)
$$

sends the closed ball of radius $r$ in $\tilde{\mathfrak{S}}_{r}\left(\oplus_{i=1}^{n} E\left(K_{i}\right), E\right)$ to itself and $\left\|N\left(A_{1}\right)-N\left(A_{2}\right)\right\|_{r} \leq \delta\left\|A_{1}-A_{2}\right\|_{r}$, for $A_{1}, A_{2}$ in the ball of radius $r$. By the contraction mapping theorem, there exists $A$, unique in $\tilde{\mathfrak{S}}_{r}\left(\bigoplus_{i=1}^{n} E\left(K_{i}\right), E\right)$, which is solution of equation (5.5).

THeOReM 5.3. Suppose that $G$ is either $P$ or $T_{4}$ and that $(S, E)$ is an analytic representation of $G$ in $E$ (for this notion see [1, Definition 2]), $S_{g}=\Sigma_{n \geq 1} S_{g}^{n}, S_{g}^{n} \in \mathfrak{R}_{n}(E)$, such that

1. $S_{g}^{1}=\left.U\right|_{G}$.

2. For any $i, j$ such that $m_{i} \neq 0$ and $m_{j} \neq 0$, then $m_{i}+m_{j}>m_{k}$, for any $k, i, j, k \in\{1, \ldots, n\}$.

Then there exists a unique $A \in \mathrm{t}\left(E_{\tau}, E\right)$ such that

$$
\left((I+A) S_{g}^{1}-S_{g}(1+A)\right) P_{\tau}^{ \pm}=0
$$

and, given $K_{i} \subset M\left(m_{i}, \varepsilon_{i}\right)$ compact $(i=1, \ldots, n)$ there exists $r>0$ such that $A \in \tilde{\mathfrak{S}}_{r}\left(\bigoplus_{l=1}^{n} E\left(K_{i}\right), E\right)$.

Proof. From [1, Proposition 5], there exists $B=\Sigma_{n \geq 2} B^{n}$ in $\mathfrak{S}_{\lambda}(E)$, for some $\lambda>0$, such that $V_{g}=(I+B)^{-1} S_{g}(I+B)$ is a smooth representation of $G$ in $E$.

By Proposition 5.1, there exists $C=\Sigma_{n \geq 2} C^{n}$ in $\mathrm{t}\left(E_{\tau}, E_{\tau}\right)$ such that

$$
\left((1+C) S_{g}^{1}\right) P_{\tau}^{ \pm}=V_{g}(I+C) P_{\tau}^{ \pm}
$$

and there exists $\lambda>0$ such that $C \in \tilde{\mathfrak{E}}_{\lambda}\left(\oplus_{i=1}^{n} E\left(K_{i}\right), E\right)$. Define $(I+D)$ $=(I+B)(I+C)$. Obviously $(I+D) S_{g}^{1} P_{\tau}^{ \pm}=S_{g}(1+D) P_{\tau}^{ \pm}$. Define now $A^{k} \in \mathfrak{R}_{k}\left(E_{\tau}, E\right)$ by

$$
\begin{aligned}
A^{k} P_{\tau}^{m_{t_{1}}, \varepsilon_{t_{1}}} \otimes \cdots \otimes & P_{\tau}^{m_{t_{k}}, \varepsilon_{t_{k}}} \\
& =D^{k} P_{\tau}^{m_{t_{1}}, \varepsilon_{i_{1}}} \otimes \ldots \otimes P_{\tau}^{m_{t_{k}}, \varepsilon_{t_{k}}} \quad \text { if } m_{i_{1}} \neq 0, \ldots, m_{i_{k}} \neq 0
\end{aligned}
$$

and $\varepsilon_{i_{1}}=\cdots=\varepsilon_{i_{k}}$ and by $A^{k} P_{\tau}^{m_{l_{1}}, \varepsilon_{i_{1}}} \otimes \cdots \otimes P_{\tau}^{m_{i_{k}}, \varepsilon_{i_{k}}}=0$ otherwise. Then $A \in \mathrm{t}\left(E_{\tau}, E\right)$ and satisfies equality (5.9).

Suppose now that there exists a second series $\tilde{A}$ satisfying the conclusion of Theorem 5.3. One gets

$$
(A-\tilde{A}) S_{g}^{1} P_{\tau}^{ \pm}=\left(S_{g}(1+A)-S_{g}(1+\tilde{A})\right) P_{\tau}^{ \pm} .
$$

Identifying degree by degree in this equality, one gets inductively $(A-\tilde{A}) P_{\tau}^{ \pm}=0$ and therefore, since $A, \tilde{A} \in \mathrm{t}\left(E_{\tau}, E\right), A=\tilde{A}$. 
6. Application to a family of wave equations. We shall now give an example describing a spinless field of mass $m>0$. One denotes by $f \rightarrow \hat{f}$ the Fourier transform in the space $\mathcal{S}^{\prime}\left(\mathbf{R}^{3}\right)$ of tempered distributions on $\mathbf{R}^{3}$. Choose a Lorentz basis $\left(X_{0}, X_{1}, X_{2}, X_{3}\right)$ in $\mathrm{t}_{4}$. One introduces the following spaces.

(1) $H_{0}$ is the space of tempered distributions, the Fourier transform of which are elements of $L^{1}\left(\mathbf{R}^{3}\right)$. If $f \in H_{0}$, define $\|f\|_{0}=\|\hat{f}\|_{L^{1}\left(\mathbf{R}^{3}\right)}$.

(2) $H_{1}$ is the space of tempered distributions $f \in \mathcal{S}^{\prime}\left(\mathbf{R}^{3}\right)$, the Fourier transform of which, $\hat{f}$, is a function such that $\vec{p} \rightarrow\left(m^{2}+|\vec{p}|^{2}\right)^{-1 / 2} \hat{f}(\vec{p})$ is in $L^{1}\left(\mathbf{R}^{3}\right)$. If $f \in H_{1}$ define

$$
\|f\|_{1}=\int_{\mathbf{R}^{3}}\left(m^{2}+|\vec{p}|^{2}\right)^{-1 / 2}|\hat{f}(\vec{p})| d \vec{p}
$$

(3) $\tilde{H}$ is the Fréchet space of functions $f$ such that the function $\vec{p} \rightarrow\left(m^{2}+|\vec{p}|^{2}\right)^{n} \hat{f}(\vec{p})$ is in $L^{1}\left(\mathbf{R}^{3}\right)$ for any $n \geq 0$.

Suppose now given an analytic mapping $J \in \tilde{\mathfrak{S}}_{r}(\mathbf{C})(r>0)$. If $\varphi \in H_{0}$, define $(J(\varphi))(\vec{x})=J(\varphi(\vec{x}))$ and $\hat{J}(\hat{f})=J(f)$. Since $L^{1}\left(\mathbf{R}^{3}\right)$ is a convolution algebra, $J$ can be considered as an element of $\tilde{\mathfrak{F}}_{r}\left(H_{0}\right)$. tion)

Consider now the wave equation (where $J(0)=J^{\prime}(0)=0$ by defini-

$$
\square \varphi_{t}+m^{2} \varphi_{t}=J\left(\varphi_{t}\right)
$$

Write $\dot{\varphi}_{t}=d / d t \varphi_{t}$. One wishes to solve (6.1) for $\varphi_{t} \in H_{0}, \dot{\varphi}_{t} \in H_{1}$ (i.e. one takes $H_{0} \oplus H_{1}$ as space of initial conditions). Define

$$
\begin{gathered}
a_{t}^{+}(\vec{p})=\hat{\dot{\varphi}}_{t}+i\left(m^{2}+|\vec{p}|^{2}\right)^{1 / 2} \hat{\varphi}_{t} \text { and } \\
a_{t}^{-}(\vec{p})=\hat{\dot{\varphi}}_{t}-i\left(m^{2}+|\vec{p}|^{2}\right)^{1 / 2} \hat{\varphi}_{t} \quad\left(i^{2}=-1\right) .
\end{gathered}
$$

Equation (6.1) is then equivalent to the system

$$
\left\{\begin{array}{l}
\frac{d a_{t}^{+}}{d t}=i\left(m^{2}+|\vec{p}|^{2}\right)^{1 / 2} a_{t}^{+}+G_{1}\left(a_{t}^{+}, a_{t}^{-}\right) \\
\frac{d a_{t}^{-}}{d t}=-i\left(m^{2}+|\vec{p}|^{2}\right)^{1 / 2} a_{t}^{-}+G_{2}\left(a_{t}^{+}, a_{t}^{-}\right)
\end{array}\right.
$$

with

$$
G_{1}\left(a^{+}, a^{-}\right)=G_{2}\left(a^{+}, a^{-}\right)=\hat{J}\left(-\frac{i}{2}\left(m^{2}+|\vec{p}|^{2}\right)^{-1 / 2}\left(a^{+}-a^{-}\right)\right) .
$$


Put $G=\left(G_{1}, G_{2}\right)$. The functions $\vec{p} \rightarrow\left(m^{2}+|\vec{p}|^{2}\right)^{-1 / 2} a_{t}^{ \pm}(\vec{p})$ belong to $L^{1}\left(\mathbf{R}^{3}\right)$, therefore $G \in \tilde{\mathfrak{S}}_{r}\left(H_{0} \times H_{0}\right)$. One now defines $E(M(m, \varepsilon))=$ $L_{d \nu}^{1}(M(m, \varepsilon))$. We can consider $a_{t}^{ \pm}$as an element of $E(M(m, \pm 1))$ by the identification $a^{ \pm}(\vec{p})=a^{ \pm}(p)$ if $p=\left(p_{0}, \vec{p}\right) \in M(m, \pm 1)$. We now denote $S^{1}=U^{m,-1} \oplus U^{m,+1}$ the representation of the Poincare group $P$ on $E=E(M(m,-1)) \oplus E(M(m,+1))$. The system (6.2) becomes

$$
\frac{d}{d t} \psi_{t}=d S_{X_{0}}^{1} \psi_{t}+G\left(\psi_{t}\right)
$$

where $\psi_{t}=\left(a_{t}^{-}, a_{t}^{+}\right)$.

If $X=\sum_{\mu=0}^{3} \alpha^{\mu} X_{\mu}$, one defines

$$
\theta_{X} \psi=d S_{X}^{1} \psi+\alpha^{0} G(\psi)
$$

which is an analytic representation of $t_{4}$ in $E_{\tau}$ compatible with $S^{1}$ (for this notion see [1, Definition 8]). By [1, Proposition 10], there exists a unique analytic representation $(S, E)$ of $T_{4}$ in $E$ such that $d S=\theta$. It results from Theorem 5.3 that there exists $A \in \tilde{\widetilde{F}}\left(E_{\tau}, E\right)$ such that given $K_{-}$(resp. $\left.K_{+}\right)$compact in $M(m,-1)(\operatorname{resp} . M(m,+1))$,

$$
A \in \tilde{\mathfrak{S}}_{\lambda}\left(E\left(K_{-}\right) \oplus E\left(K_{+}\right), E\right) \text { for some } \lambda>0,
$$

and such that

$$
(I+A) S_{g}^{1} P_{\tau}^{ \pm}=S_{g}(I+A) P_{\tau}^{ \pm} .
$$

Take $h \in E\left(K_{-}\right) \cup E\left(K_{+}\right)$with $\|h\|<\lambda$. Define $\psi_{t}=(I+A) S_{\exp t X_{0}}^{1} h$. Since $S_{g}^{1}$ is norm preserving and $S_{g}(I+A) h=(I+A) S_{g}^{1} h$, the mapping $t \rightarrow \psi_{t}$ is $C^{\infty}$ from $\mathbf{R}$ to $E$ and we have $d / d t \psi_{t}=\theta_{X_{0}} \psi_{t}$.

Coming back to equation (6.1) one gets in particular:

Proposition 6.1. There exists $A \in \tilde{\mathfrak{F}}\left(\tilde{H} \times \tilde{H}, H_{0} \times H_{1}\right)$ with the following properties:

(1) Given any compact $K$ in $\mathbf{R}^{3}$ there exists $\lambda>0$ for which $A \in$ $\tilde{\mathfrak{S}}_{\lambda}\left(H_{K} \times H_{K}, H_{0} \times H_{1}\right), H_{K}$ being the space of such functions in $H_{0}$, the Fourier transform of which vanish in the complement of $K$.

(2) If $h_{0} \in H_{K},\left\|h_{0}\right\|<\lambda / 2$ and $\hat{h}_{1}(\vec{p})= \pm i\left(m^{2}+|\vec{p}|^{2}\right)^{1 / 2} \hat{h}_{0}(p)$, equation (6.1) has a solution $\varphi_{t}$ for all $t$, with initial condition $\left(\varphi_{0}, \dot{\varphi}_{0}\right)=$ $(I+A)\left(h_{0}, h_{1}\right)$.

The approach followed in this article can be applied to systems of relativistic evolution equations with arbitrary spin with an analytic interaction (provided that the masses satisfy the inequalities given at point 2 of Theorem 5.3.) to get global solutions for small data in some sectors. 
Let us now compare the example given above with previous results. The problem of existence of global solutions for relativistic wave equations, seen from an abstract point of view, was initiated by I. Segal [11]. An example which has been studied extensively is the equation

$$
\square \varphi+m^{2} \varphi=\lambda \varphi^{p} \quad(p \in \mathbf{N})
$$

for which global solutions exist for small initial data (in a suitable Sobolev space) [10, Theorem 21], when $p \geq 3$. Furthermore, (6.3) has global weak solutions for any initial data, with the restriction $\lambda<0, p$ odd [12].

Concerning the equation $\square \varphi+m^{2} \varphi=J(\varphi)$ with $\varphi$ real, $J$ being a continuous real valued function satisfying $\varphi J(\varphi) \leq 0$, W. Strauss [13] proved that there exist real global weak solutions for any real initial data.

Global solutions which are $C^{\infty}$ in space and time for equations of the type

$$
\square \varphi=G\left(\partial_{i} \varphi, \partial_{i} \partial_{j} \varphi\right),
$$

(where $G\left(\xi_{i}, \eta_{i j}\right)$ is a $C^{\infty}$ function in $\xi_{i}$ and $\eta_{i j}$ and vanishing for $\xi=0$ and $\eta=0$, and where the space of initial data is the space of $C^{\infty}$ functions on $\mathbf{R}^{d}, d \geq 6$ ) have been proved to exist by S. Klainerman [7] if the initial data is small enough (i.e. the $L^{1}$ and $L^{2}$ norms of the initial data and a certain number of its derrivatives are small enough).

The problem of the existence of global solutions for relativistic wave equations for initial data in some sectors is discussed in a review work of M. Reed [10].

\section{REFERENCES}

1. M. Flato, G. Pinczon, and J. Simon, Non-linear representations of Lie groups, Ann. Scient. Ec. Norm. Sup., 10 (1977), 405-418.

2. M. Flato and J. Simon, Non-linear equations and covariance, Letters Math. Phys., 2 (1977), 155-160.

3. Y_ Yang-Mills equations are formally linearizable, Letters Math. Phys., 3 (1979), $279-283$.

4. On a linearization program of non-linear field equations, Physics Letters, 94B (1980), 518-522.

$5 . \quad$ Linearization of relativistic non-linear wave equations, J. Math. Phys., 21 (1980), 913-917.

6. A. Grothendieck, Produits tensoriels topologiques et espaces nucléaires, Memoirs of the A.M.S., $n^{\circ} 16$ (1955).

7. S. Klainerman, Global existence of non-linear wave equations, Comm. on Pure and Applied Math., 33 (1980), 43-101.

8. G. Pinczon and J. Simon, Extension of representations and cohomology, Reports on Mathematical Physics, 16 (1979), 49-77.

9. H. Poincare, Oeuvres, Vol. 1.

10. M. Reed, Abstract Non-linear Wave Equations, Lecture Notes in Mathematics $n^{\circ} 507$, Springer-Verlag (1976). 
11. I. Segal, Non-linear semi-groups, Ann. Math., 78 (1963), 339-364.

12. The global Cauchy problem for relativistic scalar field with power interaction, Bull. Soc. Math. de France, 91 (1963), 129-135.

13. W. Strauss, On weak solutions of semi-linear hyperbolic equations, Anais. Acad. Brazil Ciéncias, 42 (1970), 645-651.

Received September 9, 1981 and in revised form December 18, 1981.

Laboratoire de Physique-Mathematique

Faculte des Sciences Mirande-BP 138

21004-DiJon CEDEX, France 



\title{
PACIFIC JOURNAL OF MATHEMATICS EDITORS
}

\author{
Donald BabBitT (Managing Editor) \\ University of California \\ Los Angeles, CA 90024 \\ Hugo Rossi \\ University of Utah \\ Salt Lake City, UT 84112 \\ C. C. Moore and Arthur Ogus \\ University of California \\ Berkeley, CA 94720
}

J. DugundiI

Department of Mathematics

University of Southern California

Los Angeles, CA 90089-1113

R. FINN and H. SAMELSON

Stanford University

Stanford, CA 94305

\section{ASSOCIATE EDITORS}
R. ARENS
E. F. BECKENBACH
B. H. NeUMANN
F. WOLF
K. YosHIDA

(1906-1982)

\section{SUPPORTING INSTITUTIONS}

UNIVERSITY OF ARIZONA

UNIVERSITY OF BRITISH COLUMBIA

CALIFORNIA INSTITUTE OF TECHNOLOGY

UNIVERSITY OF CALIFORNIA

MONTANA STATE UNIVERSITY

UNIVERSITY OF NEVADA, RENO

NEW MEXICO STATE UNIVERSITY

OREGON STATE UNIVERSITY

\author{
UNIVERSITY OF OREGON \\ UNIVERSITY OF SOUTHERN CALIFORNIA \\ STANFORD UNIVERSITY \\ UNIVERSITY OF HAWAII \\ UNIVERSITY OF TOKYO \\ UNIVERSITY OF UTAH \\ WASHINGTON STATE UNIVERSITY \\ UNIVERSITY OF WASHINGTON
}

The Supporting Institutions listed above contribute to the cost of publication of this Journal, but they are not owners or publishers and have no responsibility for its content or policies.

Mathematical papers intended for publication in the Pacific Journal of Mathematics should be in typed form or offset-reproduced (not dittoed), double spaced with large margins. Please do not use built up fractions in the text of the manuscript. However, you may use them in the displayed equations. Underline Greek letters in red, German in green, and script in blue. The first paragraph must be capable of being used separately as a synopsis of the entire paper. In particular it should contain no bibliographic references. Please propose a heading for the odd numbered pages of less than 35 characters. Manuscripts, in triplicate, may be sent to any one of the editors. Please classify according to the scheme of Math. Reviews, Index to Vol. 39. Supply name and address of author to whom proofs should be sent. All other communications should be addressed to the managing editor, or Elaine Barth, University of California, Los Angeles, California 90024.

There are page-charges associated with articles appearing in the Pacific Journal of Mathematics. These charges are expected to be paid by the author's University, Government Agency or Company. If the author or authors do not have access to such Institutional support these charges are waived. Single authors will receive 50 free reprints; joint authors will receive a total of 100 free reprints. Additional copies may be obtained at cost in multiples of 50 .

The Pacific Journal of Mathematıcs is issued monthly as of January 1966. Regular subscription rate: $\$ 132.00$ a year (6 Vol., 12 issues). Special rate: $\$ 66.00$ a year to individual members of supporting institutions.

Subscriptions, orders for numbers issued in the last three calendar years, and changes of address should be sent to Pacific Journal of Mathematics, P.O. Box 969, Carmel Valley, CA 93924, U.S.A. Old back numbers obtainable from Kraus Periodicals Co., Route 100, Millwood, NY 10546.

The Pacific Journal of Mathematics ISSN 0030-8730 is published monthly by the Pacific Journal of Mathematics at P.O. Box 969, Carmel Valley, CA 93924. Application to mail at Second-class postage rates is pend ing at Carmel Valley, California, and additional mailing offices. Postmaster: Send address changes to Pacific Journal of Mathematics, P. O. Box 969, Carmel Valley, CA 93924.

PUBLISHED BY PACIFIC JOURNAL OF MATHEMATICS, A NON-PROFIT CORPORATION

Copyright $(1) 1983$ by Pacific Journal of Mathematics 


\section{Pacific Journal of Mathematics}

\section{Vol. 105, No. $2 \quad$ October, 1983}

Spiros Argyros, On compact spaces without strictly positive measure . . . . . 257

Steven Robert Bell, Regularity of the Bergman projection in certain nonpseudoconvex domains ............................273

Carlos R. Borges and Gary Fred Gruenhage, Sup-characterization of

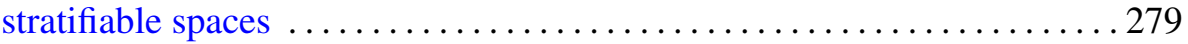

Giuseppe Ceresa and Alberto Collino, Some remarks on algebraic

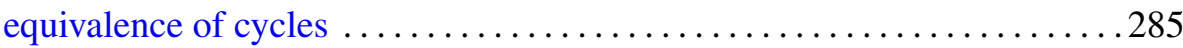

Charles Kam-Tai Chui and Maurice Hasson, Degree of uniform approximation on disjoint intervals ....................... 291

Gary Gundersen, Meromorphic functions that share two finite values with

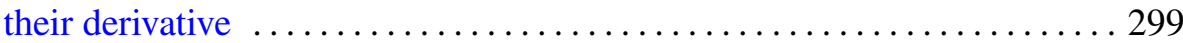

Richard I. Hartley, Lifting group homomorphisms ............... 311

Gerald William Johnson and David Lee Skoug, Notes on the Feynman integral. III. The Schroedinger equation ..................... 321

John Cronan Kieffer, Some topologies on the set of discrete stationary

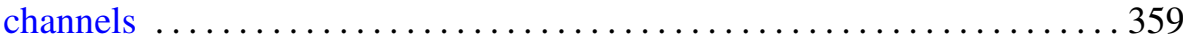

Harald Luschgy and Wolfgang Thomsen, Extreme points in the Hahn-Banach-Kantorovič setting . ............................... 387

Zbigniew Piotrowski, A. Rosłanowski and Brian M. Scott, The pinched-cube topology ................................ 399

Elias Saab and Paulette Saab, A dual geometric characterization of Banach

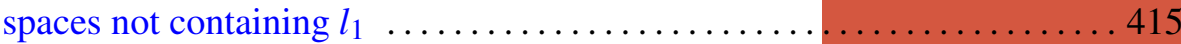

Walter Schachermayer, Norm attaining operators on some classical Banach

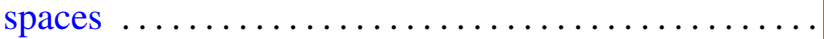

Martin Scharlemann, Essential tori in 4-manifold boundaries

Jacques C. H. Simon, Nonlinear representations of Poincaré group and global solutions of relativistic wave equations ..........

Adrian R. Wadsworth, $p$-Henselian field: $K$-theory, Galois cohomology, and graded Witt rings $\ldots \ldots \ldots \ldots \ldots \ldots \ldots \ldots \ldots$ 